\title{
Seroprevalence of Toxoplasma Gondii in Saudi Autoimmune Disease Patients
}

\author{
Ismail $\mathrm{KA}^{1,2 *}$, Zaini $\mathrm{R}^{1}$, Gharib $\mathrm{AF}^{1}$, Hagag $\mathrm{HM}^{1,3}$, Hassan $\mathrm{AF}^{1}$, \\ Alzahrani S1, Aied A1, Alhomaidi F1, Mohammed N1, Abdulaziz S1 \\ and Aljumaihe $\mathrm{S}^{1}$ \\ ${ }^{1}$ Clinical laboratory, Taif University, Egypt \\ ${ }^{2}$ Ain Shams Faculty of Medicine, Egypt \\ ${ }^{3}$ Pathology Department, Al Azhar University, Egypt
}

*Corresponding author: Khadiga Ahmed Ismail, Ain Shams Faculty of Medicine, Egypt, Email: khadigaahmed68@yahoo.com

\begin{abstract}
Toxoplasma gondii (T. gondii) is considered as one of the highly resistant intracellular parasite mainly found in the central nervous system (CNS), however, it can persist in multiple tissues in the body. Moreover, T. gondii is causing disseminated infections among patients with autoimmune diseases. Thus, the aim of this study was to evaluate the seroprevalence of toxoplasmosis in autoimmune disease Saudi patients. Ninety patients suffer from different autoimmune diseases and forty five healthy controls were subjected to determination of anti T. gondii immunoglobulin G (IgG) antibody seropositivity using commercially available Enzyme-linked Immuno sorbent assay kits (ELISA). The results showed that the seropositivity rate of anti T. gondii IgG antibodies was $22.2 \%$ the results are statistically significant. These statistically significant results support the association between T. gondii infection and different autoimmune diseases and suggest the usefulness of performing serological test for detection of infection by toxoplasma, so it is recommended early detection of Toxoplasma in autoimmune diseases patients to prevent any complications.
\end{abstract}

Keywords: Toxoplasma Gondii; Rheumatoid Arthritis; Systemic lupus Erythematous; Psoriasis; Ulcerative Colitis; Anti T. gondii immunoglobulin G

Abbreviations: ELISA: Enzyme-linked Immuno Sorbent Assay Kits; CNS: Central Nervous System; RA: Rheumatoid arthritis; SLE: systemic lupus erythematous; MS: Multiple Sclerosis; IgG: immunoglobulin G.

\section{Introduction}

Toxoplasma gondii ( $\mathrm{T}$. gondii) is an obligate intracellular protozoan parasite capable of infecting wide variety of mammals, including humans. This protozoan is 


\section{Annals of Immunology \& Immunotherapy}

able to persist in multiple tissues where the latent stage of the parasite is mainly found in the central nervous system. Although approximately $30 \%$ of the world's population has T. gondiiinfection and harbor cysts in the brain, overt disease symptoms such as encephalitis are only evident during immune suppression [1].

An autoimmune disease is a condition arising from an abnormal immune response to a normal body part. The cause is generally unknown. Some autoimmune diseases such as lupus run in families and certain cases may be triggered by infections or other environmental factors. Some common diseases that are generally considered autoimmune include celiac disease, diabetes mellitus type 1, Graves' disease, inflammatory bowel disease, multiple sclerosis, psoriasis, rheumatoid arthritis and systemic lupus erythematous [2].

Globally autoimmune diseases affect about 3\% of the population with a greater prevalence in women than men. They arise when a genetically predisposed individual with inadequate or non-functional immune regulatory mechanisms, has an immune response to an environmental pathogen [3-5].

The conventional treatment strategies to autoimmune diseases are not satisfactory [6]. The major focus of management is anti-inflammatory and immunosuppressive therapy to reduce the immune activation and thereby to reduce the inflammatory damage. Various cytokines and cells which participate in the ongoing inflammatory process are targeted. Single or combinations of immunosuppressive agents are used $[7,8]$. The aim of this study is to evaluate the seroprevalence of toxoplasmosis in autoimmune disease Saudi patients.

\section{Subjects and Methods}

Case control study was conducted from January 2018 to March 2019 on 90 ( 75 female and 15 male) patients have autoimmune diseases, blood samples were collected from the internal medicine department at King Faisal Medical Complex and King Abd Alaziz Specialized Hospital., Taif, KSA. Full clinical evaluation was done. And control group consisted of 45 healthy volunteers (24 female and 21 male), with the same range of age. The healthy control group was chosen from among the relatives/ visitors of the patients. Determination of both autoimmune markers and anti-Toxoplasma IgG by (DRG® Toxoplasma IgG (TORCH) (EIA-1798).

\section{Ethical Considerations}

The study purpose was explained to the all participants, then, an informed consent was taken from them. The study approved by the Research Ethical Committee of Applied Medical Science College, Taif University, KSA.

\section{Statistical Analysis}

Collected data were numbered, coded and introduced to a computer using the Statistical Package for Social Science for Windows version 22.0. The $\mathrm{x}^{2}$ test was used to analyze the frequency of anti $T$. gondii IgG to clarify statistically significant differences. A value of $\mathrm{P}<0.05$ was considered statistically significant.

\section{Results}

From January 2018 to March 2019, 90 autoimmune diseased patients were included from the internal medicine department at King Faisal Medical Complex and King Abd Alaziz Specialized Hospital. Patients were aged between 20 to 50 years and the twenty of them were positive for toxoplasmosis in different age groups as shown in Figure1. The distribution of toxoplasmosis among different autoimmune disease patients as shown in Figure 2. The seroprevalence of Toxoplasma among autoimmune disease patients was $22.2 \%$ as in Table 1 .

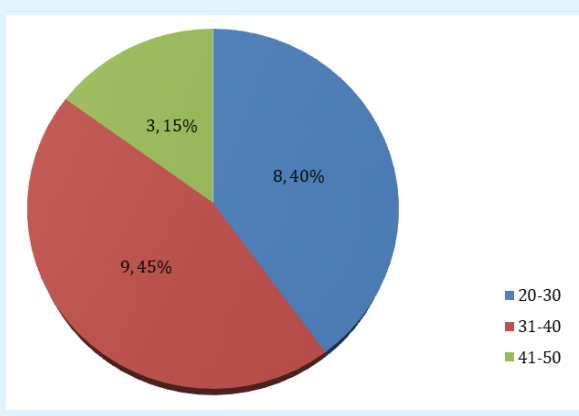

Figure 1: Number of diagnosed Toxoplasmosis in patients in different age groups. 


\section{Annals of Immunology \& Immunotherapy}

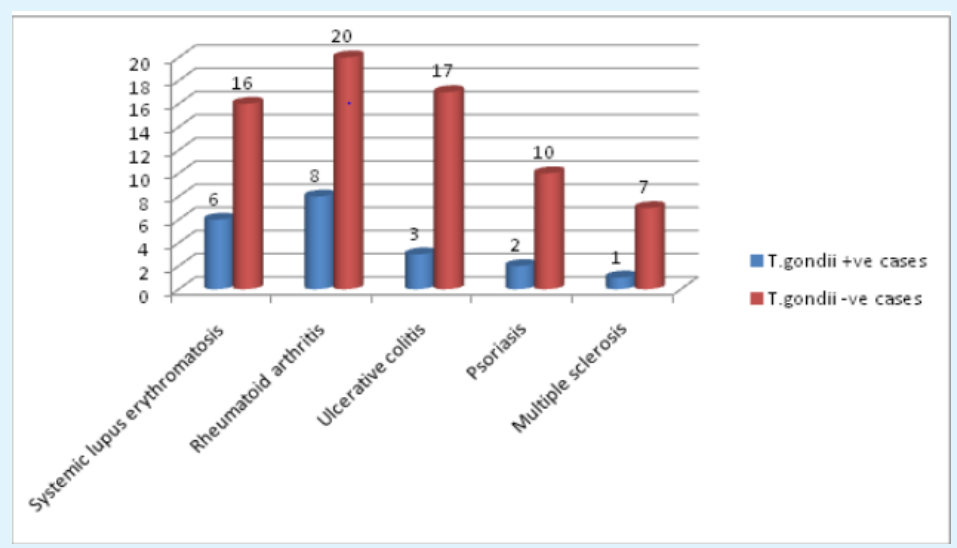

Figure 2: The distribution of toxoplasmosis among different autoimmune disease patients.

\begin{tabular}{|c|c|c|c|c|c|}
\hline & $\begin{array}{c}\text { T. gondii positive } \\
\text { № \% }\end{array}$ & $\begin{array}{c}\text { T. gondii negative } \\
\text { № \% }\end{array}$ & Total № \% & X & P-Value \\
\hline $\begin{array}{c}\text { Autoimmune } \\
\text { diseases group }\end{array}$ & $20(22.2 \%)$ & $70(77.8 \%)$ & $90(100 \%)$ & \multirow{2}{*}{9.601} & $<0.001^{*}$ \\
\hline Control group & $1(2.2 \%)$ & $44(97.8 \%)$ & $45(100 \%)$ & \\
\hline
\end{tabular}

Table 1: Toxoplasmosis seropositivity in autoimmune disease patients and control group.

Autoimmune diseases group vs control group

* Highly significant difference.

\section{Discussion}

Autoimmunity is the failure of self-tolerance processes. When the specific immune receptors fail to differentiate self from foreign, the effector mechanisms are directed toward the individual's own tissues, and pathological autoimmune disease results [9].

Toxoplasma gondii is an obligate intracellular parasite. Following oral infection the parasite crosses the intestinal epithelial barrier to disseminate throughout the body and establish latent infection in central nervous tissues. The clinical presentation ranges from asymptomatic to severe neurological disorders in Immuno compromised individuals. Since the clinical presentation is diverse and depends, among other factors, on the immune status of the host [10].

Age group of patients under this study ranged between 20-50 years as in Figure 1 and this result goes with Amador, et al. [11]. Who found that sixty-five percent of patients with systemic lupus erythematous (SLE) start manifesting their symptoms between ages 16 and 55 . Another 20 percent manifest SLE symptom before age 16 and the remaining 15 percent after age 55 year.
Rheumatoid arthritis (RA) can begin at any age but has its peak between ages 30 and 55 years. Multiple Sclerosis (MS) usually appears between ages 20 and 40 years, and it is very rare during adolescence, This may be due to Autoimmune disease usually ranged between these ages.

Most of our patients under study were female 75 $(83 \%)$ this is go with the result obtain by Nikpour, et al. [12]. Who found that autoimmune diseases is prevalent in female than male.

Eight Toxoplasma infected case within thirty RA patients included in our study As in Figure 2. this in agreement with study done by El-Henawy, et al. [13]. Who detect anti-Toxoplasma antibodies in Egyptian rheumatoid arthritis patients higher seroprevalence of anti-Toxoplasma $\mathrm{IgG}$ antibodies among RA patients compared to controls reflects an association between latent Toxoplasma infection and RA. Also six Toxoplasma infected case within twenty SLE patients included in our study this in agreement with study done by El-Henawy, et al. [13] who showed that most patients with systemic lupus erythematosus who developed toxoplasma encephalitis were misdiagnosed and treated as neuropsychiatric systemic lupus erythematosus, which 


\section{Annals of Immunology \& Immunotherapy}

led to unfavorable outcomes. They describe disseminated toxoplasmosis affecting some organs such as brain, lung and eyes with atypical symptoms, which developed with exacerbation of systemic lupus erythematosus. The initial manifestation with retinochoroiditis without vitritis, mild cognitive impairment and an isolated lung mass.

\section{Conclusion and Recommendation}

Toxoplasma gondii is an opportunistic parasite that flares up immunocompromised patients lead to encephalitis, so we recommended early detection of Toxoplasma in autoimmune diseases patients to prevent any complications. Also we recommend to increase sample size and future large-scale studies to elucidate the exact role of Toxoplasma whether a tiger of autoimmunity in autoimmune diseases or an effect of immunosuppression.

\section{References}

1. El-Sayed NM, Ismail KA, Badawy AF, Elhasanein KF (2016) In vivo effect of anti-TNF agent (etanercept) in reactivation of latent toxoplasmosis. J Parasit Dis 40(4): 1459-1465.

2. Hohlfeld R, Dornmair K, Meinl E, Wekerle H (2016) The search for the target antigens of multiple sclerosis, part 1: autoreactive CD4+ T lymphocytes as pathogenic effectors and therapeutic targets. The Lancet Neurol 15(2): 198-209.

3. Kukko M, Virtanen SM, Toivonen A, Simell S, KorhonenS, et al. (2004) Geographical variation in risk HLA-DQB1 genotypes for type 1 diabetes and signs of beta-cell autoimmunity in a high-incidence country M. Diabetes Care 27(3): 676-681.

4. Cooper G, Stroehla B (2003) The epidemiology of autoimmune diseases. Autoimmun Rev 2(3): 119-125.
5. Marrack P, Kappler J, Kotzin LB (2001) autoimmune disease: why and where it occurs. Nat Med 7(8): 899905.

6. Van der Kooij SM, de Vries- Bouwstra JK, GoekoopRuiterman YPM, Van Zeben D, Kerstens PJSM, et al. (2007) Limited efficacy of conventional DMARDs after initial methotrexate failure in patients with recent onset rheumatoid arthritis treated according to the disease activity score. Ann Rheum Dis 66(10): 1356-1362.

7. Dalakas MC, Hohlfeld R (2003) Polymyositis and dermatomyositis. The Lancet 362(9388): 971-982.

8. Dalakas MC (2006) Therapeutic targets in patients with inflammatory myopathies: Present approaches and a look to the future. Neuromuscul Disord 16(4): 223-236.

9. Eisenberg R (2003) "Mechanisms of Autoimmunity". Immunologic research 27(2-3): 203-217.

10. Munoz M, Liesenfeld O, Heimesaat M (2011) Immunology of Toxoplasma gondii. Immunol Rev 240(1): 269-285.

11. Ahmadpour E, Daryani A, Sharif M, Sarvi S, Aarabi M, et al. (2014) Toxoplasmosis in immunocompromised patients in Iran: a systematic review and metaanalysis. J Infect Dev Ctries 8(12): 1503-1510.

12. Nikpour M, Bridge J, Richter S (2014) A systematic review of prevalence, disease characteristics and management of systemic lupus erythematosus in Australia: identifying areas of unmet need. Intern Med J 44(12a): 1170-1179.

13. El-Henawy A, Hafez E, Nabih N, Shalaby N, Mashaly M (2017) Anti- Toxoplasma antibodies in Egyptian rheumatoid arthritis patients. Rheumatol Int 37(5): 785-790. 